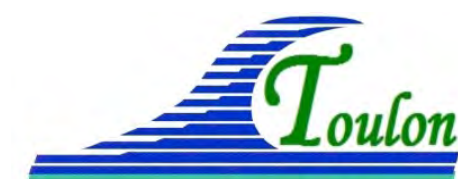

\title{
Phénomène d'amplification hydrodynamique à la culée de la grande digue flottante de Monaco : comparaisons modélisations physiques et numériques, conséquence de la mise en place d'un batardeau
}

- LECTURE SPECIALE -

\section{Christian RAFFOURT ${ }^{1}$, Philippe BARDEY ${ }^{1}$, Sacha TOUATI ${ }^{2}$, Dominique RETOURNE ${ }^{3}$}

\section{ACRI-IN, 260 Route du Pin Montard, BP 234, 06904 Sophia, France.}

Christian.raffourt@acri-in.fr ; philippe.bardey@acri-in.fr

2. Service des Travaux Publics de la Principauté de Monaco, Centre Administratif

8 rue Louis Notari, MC 98000, Principauté de Monaco.

3. NFM-Technologies, Le Lugdunum, 5 place J. Ferry, 69456 Lyon, France.

\section{Résumé :}

Le phénomène de résonance apparu à l'enracinement de la grande digue flottante de Monaco est reproduit numériquement par la méthode des singularités de Rankine. Une analyse phénoménologique explique les situations de résonance. La non linéarité de la dissipation visqueuse est prise en compte dans un processus itératif. Les résultats du modèle numérique sont comparés à ceux d'une campagne de mesures en bassin hydrodynamique. L’influence sur le phénomène de résonance de la présence d’un batardeau inséré entre la digue et sa culée est examinée sur la base de simulations numériques.

\section{Mots-clés :}

Génie côtier, Hydrodynamique maritime, Travaux maritimes.

\section{Abstract:}

Resonance phenomena appearing at Monacan big floating seawall's establisement is digitally reproduced by Rankine's singularities method. A phenomenologic analysis explains resonance situations. The non linearity of viscous dissipation is taken in account in an iterative process. The results of the digital model are compared to the ones of measures compaign in a hydrodynamic basin. The influence of the presence a inflatable barrier inserted between the seawall and its abutment on the resonance phenomena is examined on the basis of digital simulations.

\section{Introduction}

Depuis l'été 2002, la plus grande digue flottante jamais construite est ancrée à Monaco. La réalisation de cet ouvrage de 352 mètres de long et 150000 tonnes est un exploit technique. Sa mise en service en 2003 a permis d'atténuer l'agitation de l'avant-port d'un facteur 5. 


\section{Lecture spéciale}

Coté large, les mouvements de la digue sont contenus par des ancrages sur chaînes. Afin de laisser la digue libre de ses mouvements de tangage, roulis et lacet, côté terre, elle est reliée à sa culée par une rotule métallique d'un peu plus de 6 mètres de diamètre. Pour que ces mouvements soit possibles sans contact des deux structures béton, un interstice de 1 mètre est laissé libre sur toute la hauteur et la largeur entre la digue et sa culée.

La rotule dont l'axe est à -8mNGF nécessite un entretien périodique dont le prochain est planifié pour l'été 2016.

Cette intervention de périodicité décennale doit être réalisée à sec et pour cela, un batardeau gonflable en forme de "U" est inséré dans l'interstice digue-culée (voir figure 1). La pression de gonflage du batardeau permet d'assurer l'étanchéité pour que l'intérieur du "U" puisse être vidangé. Le dimensionnement de cet élément assure que l'effort d'écartement dû au gonflage soit approximativement compensé par le déficit de pression hydrostatique dû à la vidange.

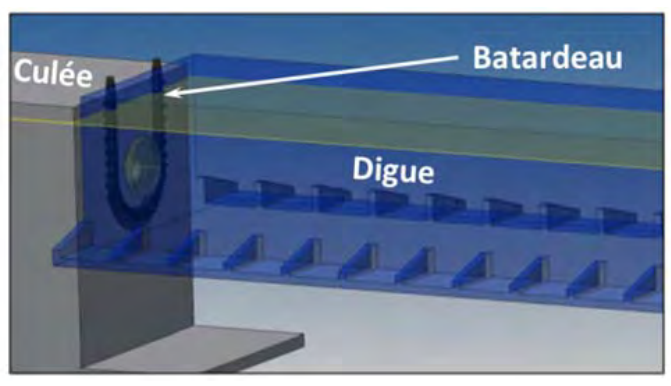

Figure 1. Localisation du batardeau

Le dimensionnement du batardeau vis-à-vis des efforts hydrodynamiques et des submersions doit prendre en compte un phénomène de résonance hydrodynamique dans l'interstice. En effet, après la mise en service de l'ouvrage, est apparu un phénomène non-anticipé d'amplification de la houle dans l'espace de l'interstice. Ce phénomène se matérialise plusieurs fois par an par des oscillations du niveau d'eau provocant des projections bien au-dessus de la dalle supérieure de l'ouvrage flottant. Plusieurs tentatives pour contenir le phénomène ont échoué. Il s'agissait d'un écran mécanique souple horizontal puis d'un panneau pare-houle vertical côté mer en vis-à-vis de l'interstice.

Le Département des Travaux Publics de la Principauté de Monaco (TPM) a chargé la société NFM Technologies de réaliser les travaux d'entretien de la rotule. NFM Technologies a sollicité la société ACRI-IN pour anticiper l'impact de la mise en place du batardeau sur les effets du phénomène de résonance hydrodynamique dans l'interstice.

La première section de ce papier présente brièvement des résultats antérieurs non publiés sur lesquels s’appuient ces travaux ainsi que les méthodes mises en œuvre pour analyser le phénomène observé et anticiper son évolution en présence du batardeau. Il 


\section{XIV èmes Journées Nationales Génie Côtier - Génie Civil \\ Toulon, 29 juin au $1^{\text {er }}$ juillet 2016}

s'agit de modélisations numériques réalisées par ACRI-IN en 2005 et de modélisations physiques conduites par Océanide en 2006. La seconde partie est dédiée à la description des résultats numériques. Elle inclut (i) une étude fréquentielle en fluide parfait, (ii) des modélisations numériques avec dissipation visqueuse comparée aux résultats de mesures disponibles et (iii) l'anticipation de l'influence hydrodynamique du batardeau. Enfin, conclusions et perspectives sont présentées dans la dernière partie.

\section{Matériel et méthodes}

\subsection{Résultats antérieurs}

\subsubsection{Les premières modélisations numériques}

La résonance hydrodynamique dans l'interstice est apparue en 2003 dès la mise en service de la digue. En 2005, afin de mieux cerner le phénomène, les TPM, avaient confié au bureau d'étude ACRI-IN, la mission de diagnostiquer l'état existant de l'enracinement de la digue d'un point de vue hydrodynamique, avant d'imaginer des solutions au stade de l'étude préliminaire. Les premières intuitions en matière d'agitation locale poussaient à rechercher l'explication des observations actuelles dans la fente en isolant uniquement les phénomènes ondulatoires instationnaires. Il était légitime de supposer qu’il s'agissait de simples résonances plutôt que de phénomènes de jets engendrés par exemple par des sous-pressions élevées sous le radier.

Il a donc logiquement été choisi de modéliser numériquement les effets hydrodynamiques tridimensionnels de diffraction et radiation aux abords proches de l'interstice. Ici, le terme diffraction traduit les perturbations sur la houle incidente induites par la présence d'une structure fixe. La radiation est associée aux ondes engendrées par les mouvements d'un corps mobile. Pour un corps libre d'osciller dans la houle, les deux phénomènes sont évidemment couplés.

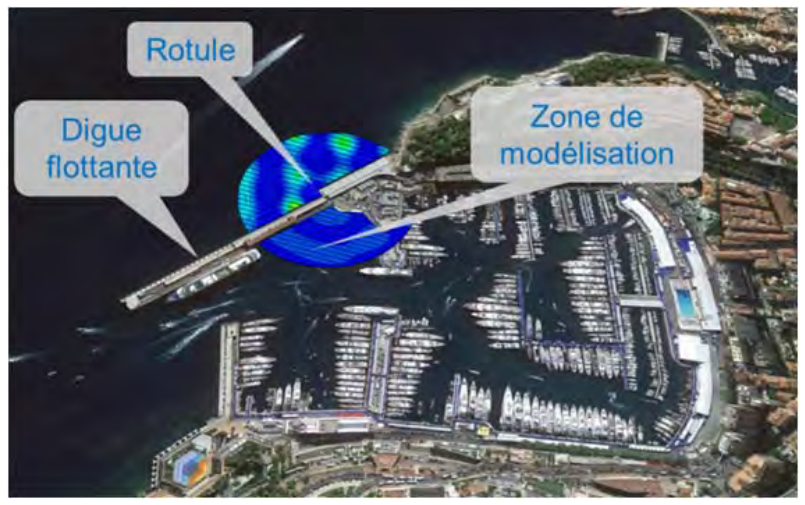

Figure 2 Localisation du modèle numérique. 


\section{Lecture spéciale}

Dans l'interstice de la digue de Monaco, les fortes oscillations observées, même par mer faiblement agitée, ne sont pas liées aux mouvements de la digue qui sont très faibles à cet endroit. La reproduction du phénomène a donc été abordée en ne simulant que la diffraction des ondes. L’emprise du modèle numérique est représentée par la figure 2.

Les hypothèses de base du modèle représentant les mouvements fluides sont les suivantes :

- l'eau de mer est assimilée à un fluide parfait et iso-volume ;

- le champ de vitesses est irrotationnel en tout point du domaine fluide (D) ;

- la condition de surface libre est la condition de Poisson linéarisée des fluides parfaits, affichée sur le plan d'eau moyen (SL), de cote $\mathrm{z}=0$;

- les caractéristiques de la houle sont supposées être celles d'une houle linéaire d’Airy ;

- la carène $(\Sigma)$ de la digue sollicitée par la houle incidente est fixe.

Il en résulte :

- d'une part, que l'écoulement qui s'établit autour de la barge peut être représenté par une fonction de potentiel harmonique $\phi(\mathrm{M}, \mathrm{t})$;

- d'autre part, que ce potentiel est une fonction sinusoïdale du temps dont la pulsation $\omega$ est égale à celle de la houle incidente.

On posera alors $\phi(M, t)=\mathfrak{R}_{e}\left(\phi(M) e^{-i \omega t}\right)$

$\phi(\mathrm{M})$ étant l'amplitude complexe de $\phi(M, t)$.

Cette notation sera généralisée à toute grandeur dépendant linéairement de ce potentiel : la pression $\mathrm{P}$, la vitesse $\overrightarrow{\mathrm{V}}$, la déformée $\eta$ de la surface libre,...; par exemple : $p(M, t)=\mathfrak{R}_{e}\left[\mathbf{P}(M) e^{-i \omega t}\right]$

Dans ces conditions, l'état du système fluide peut être représenté par le système d'équations linéaires stationnaires suivant :

$\Delta \phi=0 \quad$, en tout point du domaine fluide (D) ;

$k_{0} \phi+\frac{\partial \phi}{\partial n}=0$, en tout point de la surface libre (SL) ;

$\frac{\partial \phi}{\partial n}=0 \quad$, en tout point fixe de l'enveloppe $\partial \mathrm{D}$ de $\mathrm{D}$;

$\frac{\partial \phi}{\partial n}-\frac{\partial \phi_{I}}{\partial n}=A_{0}\left(\phi-\phi_{I}\right)+A_{2} \frac{\partial^{2}}{\partial \theta^{2}}\left(\phi-\phi_{I}\right)$, sur la frontière ouverte amont

$\frac{\partial \phi}{\partial n}=A_{0} \phi+A_{2} \frac{\partial^{2} \phi}{\partial \theta^{2}}$, sur la frontière ouverte aval

où les paramètres $A_{0}$ et $A_{2}$ s'expriment ainsi :

$$
\begin{aligned}
& A_{0}=m_{0}\left[-\lambda_{D 0}\left(1-\lambda_{D 0}{ }^{2}\right)+i\left(1+\frac{1}{2} \lambda_{D 0}^{2}\right)\right] \\
& A_{2}=\lambda_{D 0}\left(\frac{1}{R}+i m_{0}\right)
\end{aligned}
$$

avec : 


\section{XIV ${ }^{\text {èmes }}$ Journées Nationales Génie Côtier - Génie Civil \\ Toulon, 29 juin au $1^{\text {er }}$ juillet 2016}

$\lambda_{D 0}=\frac{1}{2 m_{0} R}$

$k_{o}=\omega^{2} / g$

$m_{0}$, le nombre d'ondes au niveau des frontières amont verticales,

$\vec{n}$, la demi-normale à $(\Sigma)$ qui, par convention, sera celle qui pointe vers l'extérieur du volume du domaine fluide,

$R$, le rayon de la boite de contrôle circulaire formant la frontière ouverte du domaine d'étude,

$\phi_{I}$, le potentiel complexe de la houle incidente.

Une fois déterminée la solution de ce système différentiel, on connaît le potentiel $\phi$ sur toutes les frontières du domaine. On en déduit classiquement, par application de la formule de Lagrange linéarisée, l'expression de la pression, et donc des efforts sur la structure, ainsi que l'expression des élévations de surface libre de part et d'autre de la digue et dans l'interstice.

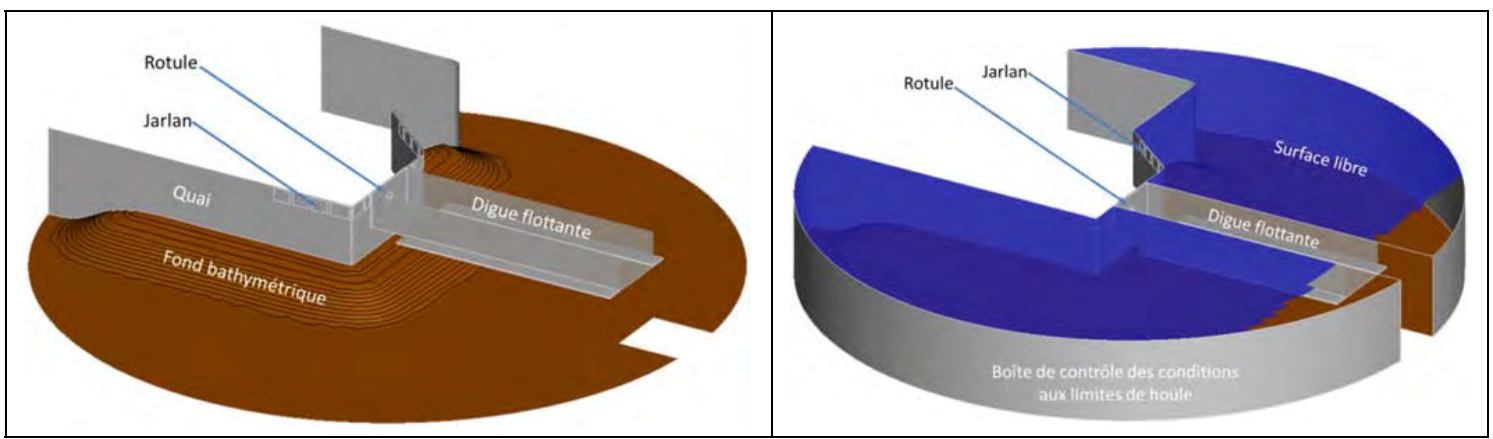

Figure 3. Schématisation des frontières physiques du problème tridimensionnel.

Discrétisation et résolution du problème. Le problème ainsi posé est résolu par la méthode des singularités de Rankine (LAJOIE, 1996). Ces singularités sont distribuées sur les frontières solides du domaine de calcul (toutes immobiles dans le cas présent). Le problème de diffraction sans vitesse d'avance en profondeur quelconque est ainsi résolu par une méthode intégrale dont les équations décrivent une distribution mixte de GREEN comportant à la fois des singularités superficielles du type sources et du type doublets normaux.

Pour $\mathrm{M}$ appartenant à la frontière $\partial \mathrm{D}$ du domaine fermé $\mathrm{D}$, il vient ainsi :

$-\frac{1}{2} \phi(M)+\int_{\partial D}\left(\phi\left(M^{\prime}\right) \frac{\partial}{\partial n_{M^{\prime}}} S\left(M, M^{\prime}\right)\right) d s\left(M^{\prime}\right)-\int_{\partial D}\left(\frac{\partial \phi}{\partial n}\left(M^{\prime}\right) S\left(M, M^{\prime}\right)\right) d s\left(M^{\prime}\right)=0$

avec $S\left(M, M^{\prime}\right)=-\frac{1}{4 \pi} \frac{1}{\left|M M^{\prime}\right|}$

Cette équation est résolue numériquement par discrétisation de la frontière $\partial \mathrm{D}$ en $\mathrm{N}$ facettes élémentaires sur lesquelles on admet que le potentiel et sa dérivée normale sont constants. Pour chaque facette $i$ de la discrétisation de $\partial \mathrm{D}$, on note $M_{i}$ son centre de 


\section{Lecture spéciale}

gravité et $\phi_{i}=\phi\left(M_{i}\right)$ le potentiel associé. Pour cette facette, l'écriture discrète de la formulation intégrale (11) s'exprime ainsi :

$\frac{1}{2} \boldsymbol{\phi}_{i}+\sum_{j \in \partial D} \boldsymbol{\phi}_{j} D_{i j}-\sum_{j \in \partial D} \frac{\partial \phi_{j}}{\partial n} S_{i j}=0$

$S_{i j}$ et $D_{i j}$ étant respectivement les coefficients d'influence des sources et doublets de la facette $j$ vers la facette $i$.

L'équation discrétisée écrite sur les $\mathrm{N}$ facettes $i$ de la frontière $\partial \mathrm{D}$ forme un système linéaire à $\mathrm{N}$ équations et $2 \mathrm{~N}$ inconnues : le potentiel et sa dérivée normale de chacune des $\mathrm{N}$ facettes. La connaissance de l'une des deux inconnues sur chaque facette de $\partial \mathrm{D}$ ou d'une relation entre les deux, rend le système colocatif, à $\mathrm{N}$ inconnues. La résolution de ce système linéaire conduit à une approche discrétisée de la solution du problème en fluide parfait.

Introduction de dissipation visqueuse. La restriction de section du mouvement fluide entrant et ressortant de l'interstice est source de dissipation visqueuse qu'il n'est pas légitime de négliger. On représente ce phénomène par une différence de pression $\Delta P$ entre l'amont et l'aval de la restriction proportionnelle au carré de la vitesse moyenne $V$ de l'écoulement :

$\Delta P=\frac{1}{2} \rho K|V| V$

où $\rho$ est la masse volumique de l'eau et où le coefficient $\mathrm{K}$ dépend principalement de la porosité équivalente représentative de la restriction de la section du jet.

En appliquant la linéarisation de Lorentz, LAJOIE 2008, propose la relation suivante entre la dérivée normale du potentiel, et ses valeurs $\phi^{+}$et $\phi^{-}$de part et d'autre de la restriction :

$$
\frac{\partial \phi}{\partial n}=\frac{\phi^{+}-\phi^{-}}{-i \frac{4 K}{3 \pi \omega}\left\{\left(-\frac{l^{2}}{\tau^{2} k_{C}^{2}}+\left[\frac{l^{4}}{\tau^{4} k_{C}^{4}}+4\left(\frac{4 K}{3 \pi \omega}\left\|\phi^{+}-\phi^{-}\right\|\right)^{2}\right]^{\frac{1}{2}}\right] / 2\left(\frac{4 K}{3 \pi \omega}\right)^{2}\right\}^{\frac{1}{2}}-\frac{l}{\tau k_{C}}}
$$

Où $\tau$ est le taux de porosité équivalente et $k_{C}$ est le coefficient de restriction de section du jet.

Cette condition est appliquée aux facettes supplémentaires d'une frontière fictive sur le pourtour de l'interstice, de façon à matérialiser la dissipation visqueuse. Initialement on suppose la vitesse nulle sur cette frontière puis la non linéarité en $\left\|\boldsymbol{\phi}^{+}-\boldsymbol{\phi}^{-}\right\|^{-1}$ est résolue itérativement. Une dizaine d'itérations est en général nécessaire pour obtenir une convergence satisfaisante. La mise en œuvre de cette technique de modélisation de la dissipation conduit à décupler des temps de calcul. 


\section{XIV ${ }^{\text {èmes }}$ Journées Nationales Génie Côtier - Génie Civil \\ Toulon, 29 juin au $1^{\text {er }}$ juillet 2016}

\subsubsection{Essais en bassin BGO FIRST}

En 2006, dans l'objectif de mieux connaître les flux existants dans l'interstice et de déterminer le type de dispositif de protection et d'atténuation le mieux adapté, les TPM ont confié à la société Océanide la réalisation d’essais sur modèle réduit dans le bassin à houle BGO FIRST. Les essais sont réalisés au $1 / 36^{\text {ème }}$ pour deux incidences de houle : Sud-Est (angle de $90^{\circ}$ avec l'axe de la digue) et Est (angle de $54^{\circ}$ avec l'axe de la digue). 14 de ces essais ont été réalisés en houles régulières et 45 en houles irrégulières.

L’instrumentation utilisée est la suivante :

- 6 sondes capacitives permettent de séparer les houles incidente et réfléchie.

- 12 capteurs de pression sont positionnés sur la culée à l'intérieur de l'interstice.

- 5 sondes résistives mesurent la surélévation locale dans l'interstice, deux autres en amont et en aval de l'interstice mesurent la houle à proximité.

Les mesures montrent l'existence de deux régimes de résonance à 4,5 secondes et 6,5 secondes (voir figure 4).

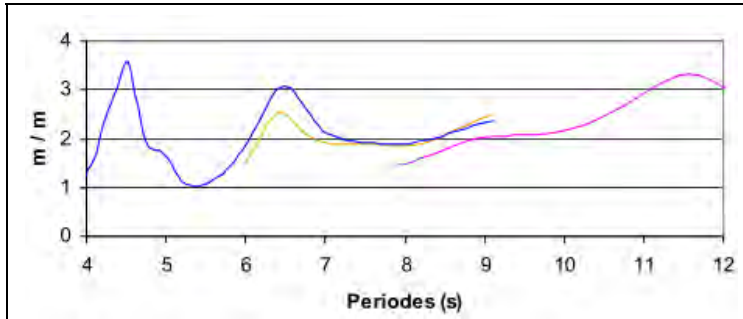

Fonction de transfert sonde RES 2

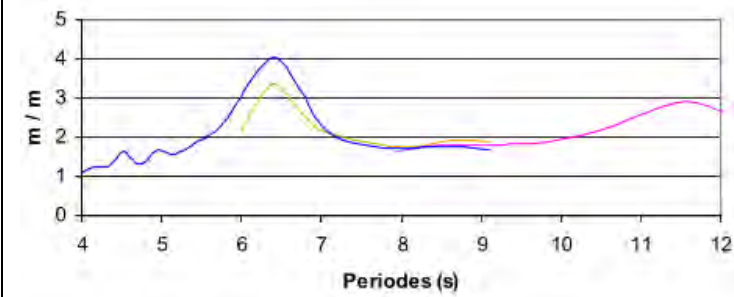

Fonction de transfert sonde RES 4

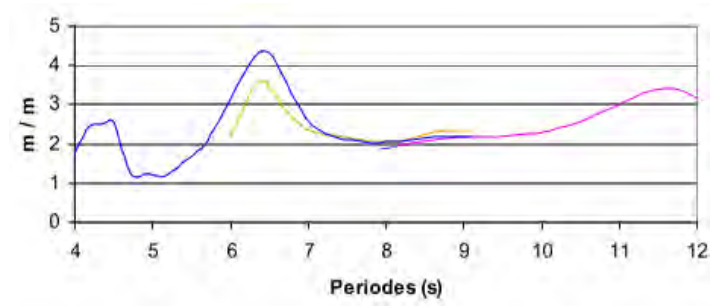

Fonction de transfert sonde RES 3

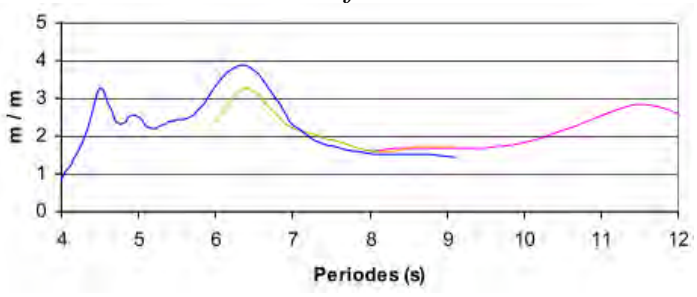

Fonction de transfert sonde RES 5

Figure 4 Fonctions de transfert entre houle dans l'interstice et houle au large d'Est.

\subsection{Méthodes}

Dans la première phase de recherche, on fait abstraction de la dissipation visqueuse afin de comprendre les mécanismes hydrodynamiques mis en jeux lors de la résonance observée dans la configuration sans batardeau. Cette analyse est conduite pour des houles d'Airy.

Les mêmes simulations sont conduites en présence du batardeau de façon à comprendre l'évolution du phénomène de résonance.

La dissipation visqueuse est ensuite activée et les résultats sont comparés aux mesures réalisées dans les mêmes conditions que celles sur modèle réduit dans le bassin BGO FIRST. Enfin, le même modèle numérique est exploité en présence du batardeau. 


\section{Lecture spéciale}

\section{Résultats}

\subsection{Modélisations numériques en fluide parfait}

Les courbes ci-après (voir figure 5) sont les fonctions de transfert entre les amplitudes des houles observées numériquement à l'intérieur et à l'extérieur de l'interstice avec et sans batardeau.



Figure 5. Amplifications maximales à l'intérieur de l'interstice par rapport à la houle à proximité.

Sur la plage de périodes 4 à 20 secondes, en l'absence de batardeau on observe deux pics de résonance, le premier à 4,5 secondes et le second à 6,2 secondes. Pour toutes les périodes de houle, l'onde est stationnaire et on constate qu'au niveau de la sortie de l'interstice l'enveloppe de phase est toujours quasi-nulle (réflexion qui se rapproche de celle d'une marche bathymétrique descendante) (voir figure 6). L'amplification trouve un premier extremum lorsque la longueur d'onde correspond à la largeur de l'interstice (voir figure 7) et un second lorsqu'elle est double (voir figure 8).

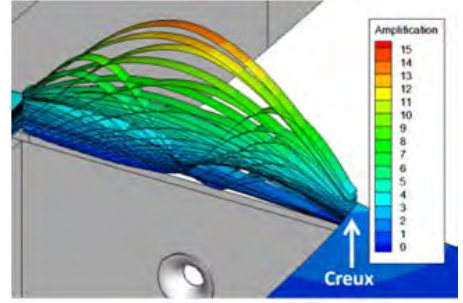

Figure 6. Enveloppes de phase positives dans

l'interstice sans batardeau pour les périodes de houle de 4 à 20 secondes.

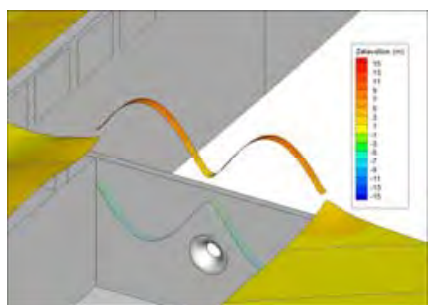

Figure 7. Enveloppe de phase dans l'interstice pour une houle de 4,4 secondes.



Figure 8. Enveloppe de phase dans l'interstice pour une houle de 6,2 secondes. 


\section{XIV èmes Journées Nationales Génie Côtier - Génie Civil \\ Toulon, 29 juin au $1^{\text {er }}$ juillet 2016}

Sur la même plage de périodes, en présence de batardeau, un seul régime de résonance subsiste. Pour toutes les houles, le maximum d'amplification est obtenu à la face du batardeau contre laquelle vient réfléchir l'onde gravitaire (voir figure 9). L'extremum d'amplification s'observe lorsque la longueur d'onde est quadruple de la distance entre l'entrée de l'interstice et la position du batardeau (voir figure 10).

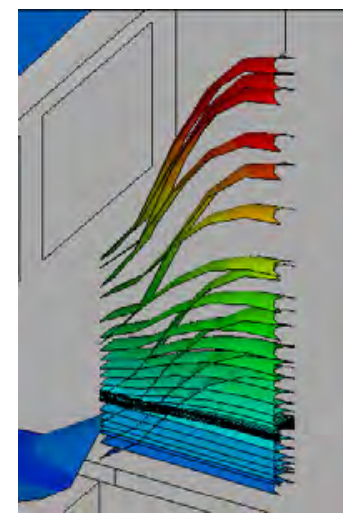

Figure 9. Enveloppes de phase positives dans l'interstice avec batardeau pour les périodes de houle de 4 à 20 secondes.

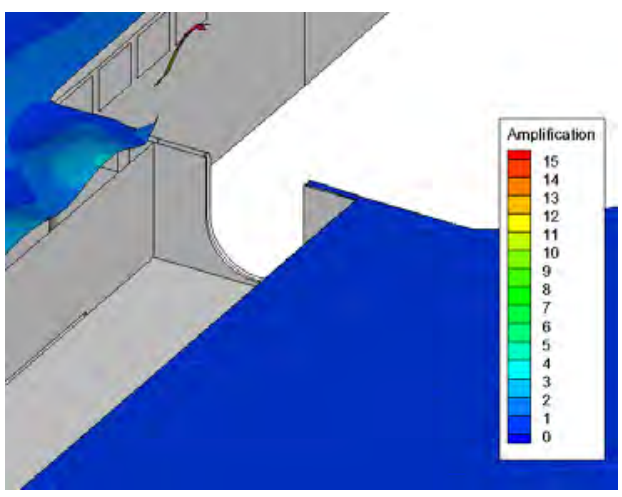

Figure 10. Enveloppe de phase positive dans l'interstice pour une houle de 4,5 secondes avec batardeau.

\subsection{Dissipation visqueuse sans batardeau}

Avec l'activation de la dissipation visqueuse, les pics de résonance sont amoindris en amplitude mais leurs largeurs de bandes sont accrues (voir figure 11). Ils sont aussi déplacés vers des périodes un peu supérieures : 4,9 secondes et 7,2 secondes. Ici, l'amplification est donnée par rapport à la houle au large.

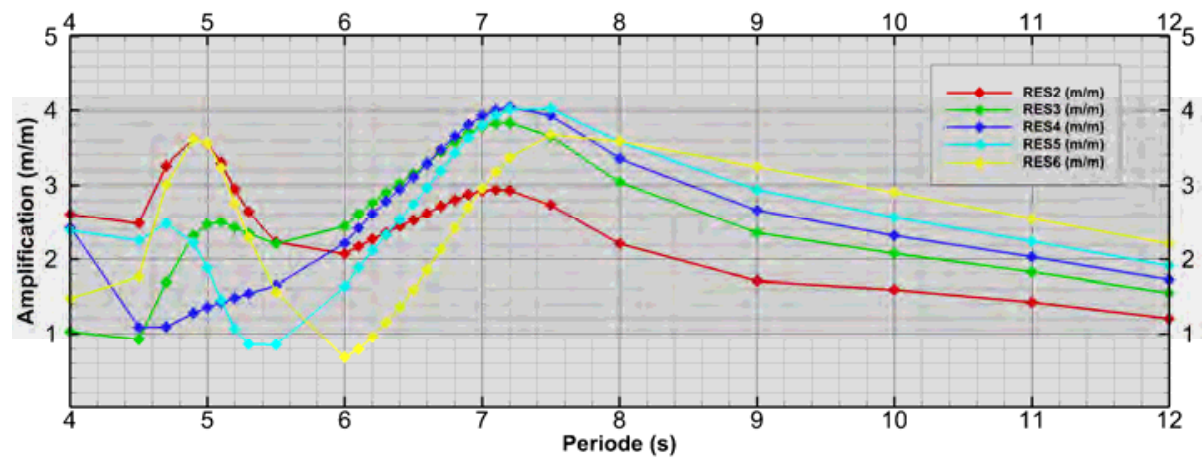

Figure 11. Graphe de l'amplification avec dissipation visqueuse pour la direction Est.

La comparaison avec les résultats des mesures d'Océanide montre que le modèle proposé permet une approche satisfaisante aux plans qualitatif et quantitatif. L'origine des imperfections est certainement majoritairement due à l'expression de K (équation 


\section{Lecture spéciale}

14) qui ne prend pas en compte la dissymétrie qui existe entre les flux entrants et sortants de l'interstice.

\subsection{Dissipation visqueuse avec batardeau}

Les paramètres de dissipation visqueuse sont conservés pour mener la modélisation en présence de batardeau. De même que sans batardeau, le pic de résonance est amoindri en amplitude avec une largeur de bande élargie.

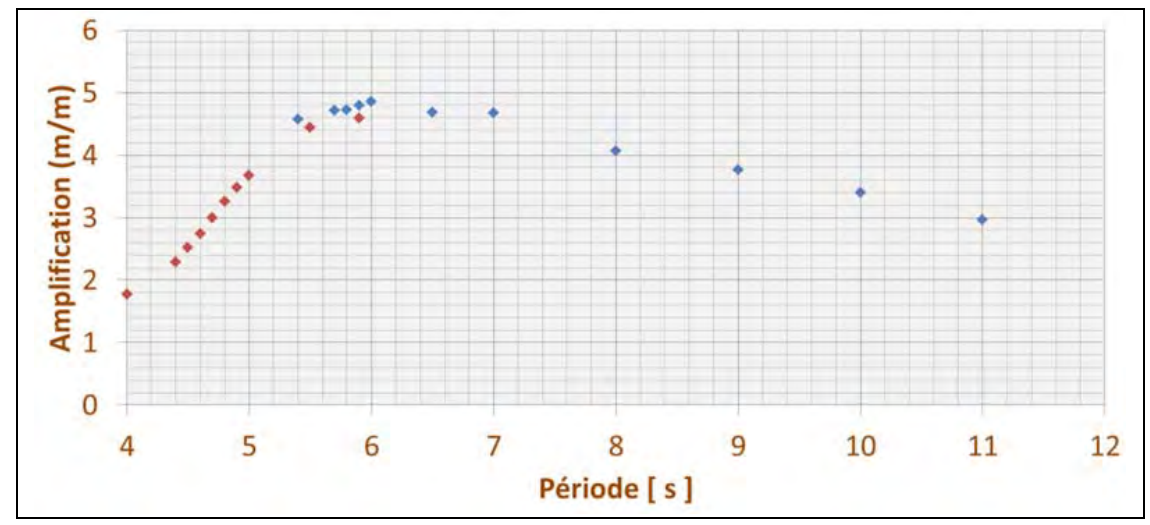

Figure 12. Graphe de l'amplification maximale dans l'interstice pour une houle d'Est.

\section{Conclusions}

Pour reproduire le phénomène de résonance observé à la culée de la grande digue flottante de Monaco, le modèle numérique SIMAC-Rankine-3D a été exploité dans sa version d'origine sans prise en compte de la dissipation visqueuse puis avec la mise en œuvre cette fonctionnalité pour les configurations avec et sans batardeau. Sans dissipation visqueuse, le modèle numérique permet d'apporter une compréhension de la phénoménologie à l'origine des résonances. L'activation de l'option de dissipation visqueuse permet d'approcher quantitativement le comportement mesuré sur modèle réduit.

L'exploitation du modèle numérique pour toutes les directions de houles permet d'anticiper le risque de submersion du batardeau.

Les résultats des modalisations numériques sans prise en compte de la dissipation visqueuse montrent bien que celle-ci a une importance primordiale dans l'évaluation des amplitudes extrêmes du phénomène. Les modélisations physiques sur modèle réduit ne pouvant parfaitement respecter simultanément la similitude de Froude et celle de Reynolds, il serait intéressant d'effectuer des mesures autour de l'ouvrage lui-même de façon à quantifier avec certitude le phénomène. Une autre piste pourrait être la réalisation d'un modèle numérique résolvant les équations de Navier-Stokes en turbulent avec prise en compte de la déformation de la surface libre par une approche 


\section{XIVìmes Journées Nationales Génie Côtier - Génie Civil \\ Toulon, 29 juin au $1^{\text {er }}$ juillet 2016}

"Volume Of Fluid" (VOF). Le balayage fréquentiel avec une telle approche, serait certainement trop coûteux en temps de calcul mais permettrait par exemple de valider un dispositif de lutte contre les résonances pour les caractéristiques de houles les plus sévères.

Le phénomène observé à Monaco trouve son origine dans la configuration particulière de l'espace confiné d'une surface libre entre deux parois verticales, parallèles et proches. Cette configuration peut apparaitre pour d'autres types d'ouvrages de façon permanente ou temporaire comme par exemple entre le quai et le portail en position ouverte d'une écluse. Pour de telles configurations, la possible apparition du phénomène de résonance peut être un argument de conception d'autant qu'outre la nuisance de l'amplification des oscillations de la surface libre, le phénomène mis en évidence a aussi un impact sur l'amplitude des contraintes hydrodynamiques qui sollicitent les éléments de liaison destinés à maintenir l'écartement entre les deux parois. Ces contraintes peuvent être non négligeables pour l'évaluation des efforts extrêmes mais aussi pour la fatigue des liaisons.

En ce qui concerne la grande digue flottante de Monaco, si ce phénomène de résonance n’était pas clairement identifié en phase de conception, les études menées à ce sujet $a$ postériori par NFM-Technologies ont montré que, compte tenu des marges de sécurité retenues, le dimensionnement de la rotule de liaison n’était pas à remettre en cause.

\section{Remerciements}

Nous remercions sincèrement le Département des Travaux Publics de la Principauté de Monaco ainsi que la société NFM Technologie et en particulier Messieurs Touati et Retourné pour la confiance qu'ils nous ont accordée.

\section{Références bibliographiques}

LAJOIE D. (1996). Modélisation de la houle en zone côtière : prévision de l'agitation à l'intérieur des ports et mise au point d'atténuateurs de houle dynamiques. Thèse de l’Université Aix-Marseille II.

LAJOIE D. (2008). Optimisation du fonctionnement des atténuateurs de houle de type "dos de chameau" à l'aide de perforations dans la structure. $\mathrm{X}^{\text {èmes }}$ Journées Nationales Génie Côtier - Génie Civil, Sophia Antipolis, pp 749-759. http://dx.doi.org/10.5150/jngcgc.2008.071-L 
Lecture spéciale 